\title{
A Qualitative Study of Breastfeeding and Formula-Feeding Mothers' Perceptions of and Experiences in WIC
}

\author{
Rebeca Almeida ${ }^{1}$; Shawnee Alvarez Gutierrez, BS $^{1}$; Shannon E. Whaley, $\mathrm{PhD}^{2}$; \\ Alison K. Ventura, PhD, CLEC ${ }^{1}$
}

\begin{abstract}
Objective: To explore Special Supplemental Nutrition Program for Women, Infants, and Children (WIC) participants' perspectives about why formula-fed infants are less likely to be recertified at 1 year compared with breastfed infants.

Design: Four focus groups of WIC mothers, stratified by language spoken (English or Spanish) and feeding mode (breastfeeding $[\mathrm{BF}]$ or formula-feeding $[\mathrm{FF}]$ ).

Setting: Two WIC sites within Los Angeles County, CA.

Participants: Mothers of 6- to 12 -month-old infants $(n=31)$ in the WIC program.

Phenomenon of Interest: Mothers' perceptions of (1) how WIC supports BF and FF mothers; and (2) experiences of FF mothers in WIC, with a focus on how these experiences may affect desire to recertify their infant in WIC at age 1 year.

Analysis: Thematic analysis of verbatim transcripts.

Results: Mothers in all focus groups discussed the perceived positive value of BF support, food assistance, nutrition education, referrals, convenient WIC services, and social support from staff. Themes related to experiences of FF mothers included feeling judged for not BF, perceptions of WIC as a formula provider, and perceived difficulties obtaining formula. Mothers indicated that these experiences affected motivations to recertify.

Conclusions and Implications: Although WIC provides important and effective support to low-income families, especially related to BF, some FF mothers may feel underserved with respect to support for their feeding decisions.
\end{abstract}

Key Words: Special Supplemental Nutrition Program for Women, Infants, and Children (WIC), breastfeeding, formula-feeding, program retention, nutrition education

\section{INTRODUCTION}

The Special Supplemental Nutrition Program for Women, Infants, and Children (WIC) is a food and nutrition assistance program designed to safeguard the health of low-income pregnant and postpartum women, infants, and children up to 5 years of age who are nutritionally at risk. ${ }^{1}$ The WIC program aims to help women and children meet their unique nutritional needs during these critical periods of growth and development. In 2017, WIC served approximately 7.3 million women, infants, and children in the US. ${ }^{2}$ In fact, half of all infants and one quarter all of children aged $1-5$ years in the US are WIC participants. ${ }^{3}$ The services provided by WIC are distributed across 4 main components: supplemental food, nutrition education, breastfeeding (BF) support, and referrals to other health

\footnotetext{
${ }^{1}$ Department of Kinesiology and Public Health, California Polytechnic State University, San Luis Obispo, CA

${ }^{2}$ Division of Research and Evaluation, Public Health Foundation Enterprises WIC, Irwindale, CA

Conflict of Interest Disclosure: The authors have not stated any conflicts of interest.

Address for correspondence: Alison K. Ventura, PhD, CLEC, Department of Kinesiology and Public Health, California Polytechnic State University, One Grand Ave, San Luis Obispo, CA 93407; E-mail: akventur@calpoly.edu
}

and social services. The effectiveness of WIC is demonstrated by previous research illustrating improved health outcomes for WIC infants, such as decreased risk for prematurity, ${ }^{4-6}$ low birth weight, and anemia. ${ }^{7,8}$

Women enrolled in the WIC program have access to BF education and support activities during their prenatal and postpartum periods. Each year, California WIC staff who provide information on $\mathrm{BF}$ to participants receive at least 4 hours of training related to the promotion and management of BF. Staff are expected to educate participants about the benefits of BF, how to breastfeed and maintain optimal milk production, and cue-based infant feeding to ensure the best health outcomes for both mothers and infants. ${ }^{9}$ In some WIC sites, BF education and support are further facilitated by peer counselors and/or lactation consultants. Both peer counselors and lactation 
consultants are resources solely available to BF WIC participants; they provide $B F$ mothers with a strong social support network wherein they can ask questions, share concerns, and receive guidance on how to reach their BF goals.

Breastfeeding is further supported by the food packages WIC provides to postpartum mothers because the amount of food included in these packages varies based on whether the mother is fully BF, partially BF, or fully formula-feeding (FF). Mothers who are fully or mostly BF are eligible to redeem an enhanced food package to support the increased caloric needs associated with lactation and receive these benefits up to 1 year postpartum. Maternal food benefits cease after 6 months postpartum for fully FF mothers because of the reduced caloric needs of the $\mathrm{FF}$ woman compared with a BF woman, and because WIC food packages include formula for the infant through 12 months of age.

Despite all these benefits, a substantial decline in WIC participation after children turn 1 year old was documented. $^{10}$ Previous research highlighted an array of barriers that may impede participants' abilities or desires to remain in the WIC program. These include participants' perceptions of the low value of food packages, ${ }^{11,12}$ eligibility restrictions, $^{13}$ stigma, $^{13,14}$ or other logistical factors such as transportation, having to bring required paperwork, and return to work or school. ${ }^{4}$ In addition to these barriers, FF was identified as a strong predictor of program attrition, as evidenced by lower rates of recertification into the program when infants turn 1 year old for FF compared with BF infants. ${ }^{10,15}$ This trend may be attributable to the discontinuance of WIC checks for formula once the child turns 1 year old, but it is also possible that mothers' experiences as a BF vs FF WIC participant may influence their propensity to remain within the WIC program. To date, few studies explored reasons for disparities in attrition for FF vs BF WIC participants. To address this research gap, the objective of this exploratory focus group study was to identify why FF mothers were less likely to recertify in the WIC program compared with BF mothers. Specific aims were to (1) determine the ways in which WIC supports both BF and FF mothers; and (2) identify mothers' perceptions of the experience of being an FF mother in WIC and evaluate how those perceptions might affect ongoing program participation.

\section{METHODS}

\section{Participants}

Participants $(n=31)$ for focus groups were recruited by WIC staff from a list of current WIC mothers who had 6- to 12-month-old infants. The study took place in 2 targeted WIC sites within Los Angeles County, CA. Specifically, to ensure that the participants were representative of the population of interest, selected sites had retention rates (defined as the percentage of eligible infants who were recertified into the program when they turned 1 year old) that fell into the lower half of WIC sites within Los Angeles County. All participants provided informed consent and received a \$20 gift card compensation for participation. Refreshments and child care were provided to facilitate the participation of mothers who had other children. Review by an institutional review board was not required to conduct these focus groups because they (1) were conducted as part of public health practice under the direct supervision of a governmental public health agency; (2) were designed to evaluate a public benefit program and service delivery; (3) were not designed to test an experimental hypothesis, drug, or device; and (4) did not collect confidential, identifiable information from participants. ${ }^{16}$ However, after completion of the focus groups and preliminary analysis of the data, the WIC evaluation team thought that the findings were impactful and merited further, more in-depth analysis by an external university collaborator (A.K.V.) and broader dissemination of the findings beyond the local agency. Before this phase of the study, the California Polytechnic State University, San Luis Obispo Institutional Review
Board reviewed and approved the analysis of these anonymous data.

\section{Focus Groups}

Previous empirical research indicated that 3 focus groups would be sufficient to achieve saturation of themes within a given population ${ }^{17}$; to this end, 4 focus groups were conducted to ensure adequate representation of WIC mothers and theoretical saturation of meaningful themes. To encourage rich conversation among mothers with similar experiences, ${ }^{18}$ interested mothers from the 2 WIC sites were purposefully organized into focus groups similar in preferred language (English or Spanish) and current feeding mode (BF, including fully or mostly BF, or FF). The composition of the 4 groups was as follows: English-speaking and BF $(n=9)$, Spanish-speaking and BF $(n=8)$, Englishspeaking and FF $(n=9)$, and Spanishspeaking and FF $(n=5)$. Focus groups with English-speaking mothers were conducted in English, and focus groups with Spanish-speaking mothers were conducted in Spanish.

Participants received a reminder call or text by a WIC staff member 1 -2 days before the focus group. This reminder call or text also confirmed the need for child care and the number of individuals intending to participate in the focus group. Focus groups were facilitated by 1 of 2 WIC research assistants; the facilitator was assisted by a notetaker and a flip chart notetaker. Both facilitators were bilingual and trained in focus group facilitation by an expert in qualitative methods. In an attempt to decrease potential response bias by participants, focus groups were facilitated by research assistants who did not regularly interact with the participants or provide WIC services to mothers.

To promote participant comfort and authenticity of responses further, the facilitators initiated each focus group by informing participants that no personally identifiable information would be used, and their responses would be kept confidential. Facilitators also assured participants that their decision to participate, as well as anything shared during the group, would not affect their receipt 
of public benefits. Facilitators led each focus group discussion using a semistructured focus group guide. The guide was adapted from focus group discussion guides developed and used in previous research with postpartum WIC mothers on the topic of gestational weight gain. ${ }^{19}$ Adaptations were informed through 1:1 interviews with a small number of postpartum WIC participants not involved in the focus groups, to ensure the questions were understandable and related to their WIC experiences. The discussion guide prompts and questions were informed by the Theory of Planned Behavior, ${ }^{20}$ which provides a framework for understanding the likelihood that an individual will engage in a health behavior. Thus, discussion guide prompts and questions were designed to understand mothers' (1) attitudes toward WIC and the services and support provided by WIC, (2) subjective norms regarding other WIC participants' experiences and perceptions of WIC, (3) perceived behavioral control related to perceived benefits that promote retention in the WIC program and perceived barriers that might deter recertification in the WIC program, and (4) intentions to continue participating in WIC and reflections on how their attitudes about and experiences with WIC might influence their intentions. In general, the focus group discussion guides were designed to explore 2 key research questions: (1) In what ways does WIC support both BF and FF mothers? (2) What are mothers' perceptions of the experience of being an FF mother in WIC, and how might these perceptions affect retention? All focus group discussions were audiorecorded to facilitate later qualitative analysis; each focus group lasted approximately 90 minutes.

\section{Data Analysis}

After each focus group session, audio recordings were transcribed verbatim and Spanish transcripts were then translated to English. Transcripts from Spanish focus groups were translated by an experienced translator who was a native Spanish speaker and was bilingual and fluent in oral and written Spanish. To ensure the confidentiality of participants, any identifying information shared during the focus groups (eg, names and ages of participants or their children) was changed to a pseudonym or deleted from the transcriptions. Deidentified transcription documents were then qualitatively analyzed using a thematic approach. ${ }^{21}$

Specifically, 3 trained coders analyzed all transcripts using constant comparison within the framework of grounded theory. ${ }^{22}$ One coder was a doctoral-level researcher with previous academic training and research experience with analyzing qualitative data; the doctoral-level researcher led the coding team and trained the other 2 coders. During the coding process, all coders coded all transcripts using the following process. First, all coders independently coded 1 transcript (BF-English) using an inductive, open coding approach in which coders identified distinct themes and subthemes that corresponded to each research question. ${ }^{21}$ Results were then reviewed and compared for validity by the coding team and any discrepancies in theme identification or coding were discussed. Coding discrepancies were minimal but were reconciled by intensive group discussion and consensus agreement on the final code. $^{23}$ Coders then independently coded the FF-English transcript using the themes and subthemes identified during the first round of coding while expanding, refining, and/or adding to these themes. Results were again reviewed and compared for validity by the coding team, and any discrepancies were discussed and reconciled by group consensus. All coders then independently coded the remaining transcripts (BF-Spanish and FF-Spanish) using the themes and subthemes identified in the first and second rounds of coding. Coders met after coding each transcript to compare results and discuss and reconcile any discrepancies in theme identification, coding, or the potential addition of new themes or subthemes. Interrater agreement was high (kappa > 0.80). After all transcripts were coded, the doctoral-level researcher (A.K.V.) rereviewed the results of all coders and further condensed themes and subthemes.
Several strategies were used to promote trustworthiness of the findings. ${ }^{24}$ To promote credibility, focus groups were purposefully conducted with BF and FF and English- and Spanish-speaking mothers in an attempt to achieve triangulation of sources. The researchers achieved analyst triangulation by having multiple coders from diverse backgrounds and experiences. Member-checking of the results was not feasible because of to varying levels of literacy in this population, but WIC staff who routinely worked closely with the population of interest reviewed and provided input regarding the validity of the themes that emerged relative to their expertise and experiences. To promote dependability and confirmability, coders engaged in a standardized process for coding and analysis, and the research team communicated often to ensure consistency and reduce bias during coding. The research team also maintained an audit trail to document analytic decisions throughout the coding process.

\section{RESULTS}

\section{In What Ways Does WIC Support Both BF and FF Mothers?}

Table 1 summarizes the themes and subthemes that emerged from the first research question on the ways in which WIC supports both BF and FF mothers and indicates the focus groups within which the themes and subthemes emerged. Major themes that emerged included (1) perceived positive value of WIC BF support, (2) material supports (eg, formula and supplemental food), (3) nutrition education and recipes, (4) referrals to other services, (5) services that increase convenience, and (6) social support provided by staff.

Breastfeeding support. Within mothers' discussion of the BF support provided by WIC, subthemes focused on the value of the $\mathrm{BF}$ counseling and encouragement provided by WIC, as well as the value of tangible support in the form of BF equipment and supplies. With respect to BF counseling and encouragement, many mothers indicated that WIC was an important source of social support for $\mathrm{BF}$ that was not available in their social 
Table 1. In What Ways Does WIC Support Both BF and FF Mothers?

\section{Themes}

BF support

\section{Subthemes}

Counseling and encouragement

Supplies

General

Provision of formula

Provision of healthy food

Nutrition education and recipes

Referrals to other services

Services that increase convenience

Hours and locations

Online education

Reminder texts and calls
Focus Group

BF-English

BF-Spanish

FF-English

FF-Spanish

BF-English

BF-Spanish

FF-English

BF-Spanish

FF-English

FF-Spanish

BF-Spanish FF-English

FF-Spanish

BF-English

BF-Spanish

FF-English

FF-Spanish

BF-English

BF-Spanish

FF-English

FF-Spanish

BF-English BF-Spanish FF-English FF-Spanish BF-English FF-Spanish

BF-English FF-English BF-English BF-Spanish FF-Spanish

\section{Key Examples}

I really enjoy the counseling for breastfeeding because being a new mom I didn't know how to breastfeed. And at the doctors, it didn't seem like they had 1-on-1 time so it really helped coming here and feeling comfortable letting them help me. That's what I like about WIC. (BF-English)

... they support you with your lactation by lending the pumps to you if you are working so that you take it to your house. (BF-Spanish)

I think apart from everything, I think it's more of the support and the services they give us. Because, like we were saying, it no longer has to come out of pocket to buy certain things. So, that's very important. Therefore, all the things they give us; more or less it's an economical support because we save ourselves a bit of money. (FF-Spanish)

Well, I have an 11-month-old baby. So, just the formula, it's like 100 and something dollars right there. So, that's a big help for me. (FF-English)

We value the food that they provide us, which is nutritious. (BF-Spanish)

Well, I come from El Salvador, and I'm going to tell you that I have a son who is 25 in EI Salvador, and I never received a class about the things I had to know for a baby. Over there, if your chest is congested, they give you honey, something that here is known to not be good for babies. It's an education that we receive and it benefits us a lot. It makes the children grow better because I notice it with my daughter. It is very important for me. (FF-Spanish)

I really appreciate the referrals. Referrals to MediCal, food stamps program, all that we need. Because we know that financial issue is really real in our situation right now. So, support in nutrition, breastfeeding, and referrals to other agencies; that's really important. (BF-English)

I work full time. And, sometimes I work overtime. So, sometimes I miss the WIC hours. So, it's easier for me to come in on Saturday when you guys are open. I think it's 1 Saturday in the month, or 2. So, it really helps out, even that 1 Saturday. (BF-English)

I think you guys improved so much already. Because now with the classes we do them through our phone, computers, you know. (FF-English)

I also like the fact that they call, because sometimes our mind is elsewhere. They remind you that you have to pick up your coupons. Like, it's something good for me, it's very nice. (FF-Spanish) 
networks. In addition, several mothers indicated that they had no knowledge or prior experience with BF; thus, WIC provided education and counseling that was critical for successfully initiating and maintaining BF. For example, 1 mother indicated:

... [The WIC program] really helped me because it was my firsttime breastfeeding and I grew up in foster care so I didn't really have a support system to teach me about breastfeeding because no one I knew did it. So, coming here really helped. (BF-English)

With respect to BF supplies and equipment, mothers discussed their appreciation for the provision of pumps and pump accessories, which also supported mothers' BF abilities.

Material supports (eg, formula and supplemental food). A robust theme across all groups was appreciation for the material support WIC provided, with subthemes focused on general material support and material support through the provision of formula and healthy foods. With respect to general material support, mothers broadly indicated that the material support WIC provided allowed them to concentrate their limited resources on other financial demands, such as rent or utilities. For example, 1 mother indicated:

The way we save money is by receiving milk, Gerber for the children, and the cereal ... we are the lower class ... it's something very beneficial for our family. (FF-Spanish)

Mothers in both of the FF focus groups, as well as 1 mother in the BFSpanish focus group, discussed an appreciation for the formula provided by WIC. Most recognized that formula is an expensive product and indicated that it would be burdensome to purchase it without WIC support. Similarly, mothers in all groups indicated that they valued the food provided within the WIC packages, with a specific focus on their appreciation for the healthfulness of the foods provided. Several mothers expressed their desire to eat healthfully and provide healthful foods for their family, and they indicated that WIC supported those goals. As 1 mother explained:
I believe that nutritious food, even though we sometimes already know what we should eat, whenever we come [to WIC], it is a reminder. It's not a bad thing for someone to continuously remind you. That's good. The reminder of nutritious food. (BF-Spanish)

Nutrition education. In a related theme, mothers in all groups also discussed that they valued the nutrition education and recipes provided by WIC. Mothers indicated that this information was particularly helpful with their first child and when they had a child after a long gap in childbearing. They acknowledged that recommendations were always changing, and WIC helped them feel confident they were engaging in healthy practices and caring for their children in ways that adhered to current recommendations. For example, 1 mother indicated:

The pamphlets, you know you guys give all that stuff when we come in. Just a lot of good information on how to eat right: ideas, how to stay healthy while you're pregnant, ideas on what to give your son. You know, like they would tell me because I didn't know how to feed him. (FF-English)

Referral to other services. Two other themes focused on aspects of WIC that helped families streamline their experiences, such as referrals to other services and WIC services that made their experience more convenient. Across all focus groups, mothers discussed how, at some point in their history with WIC, a WIC staff member had understood their needs and referred them to an appropriate service beyond WIC. These referrals ranged from other federal assistance programs to medical specialists. As shared by 1 mother:

The information they give you, because when my son was born, he had a speech delay, which I thought he had autism. And they gave me information on where I could take him you know to see what was going on with him.... So the information that I got from here, I was able to take my son there. (FF-English) 
Services that increase convenience. Mothers indicated that they valued the fact that WIC offered conveniences such as longer office hours, Saturday hours, centrally located offices, opportunities for online education, and reminder texts and calls. Several mothers discussed how these options allowed them to access WIC services without having to compromise their work and familial responsibilities.

Social support provided by staff. Mothers in all focus groups also emphasized how much they valued the social support provided by WIC staff. Many perceived the staff to be kind and caring. Many also cited experiences in which the staff made them feel genuinely supported and cared for. For example, 1 mother indicated:

Kindness, because you do not want to go to a place that makes you feel bad, but here they make you feel good. And there are times that sometimes you come crushed with whatever is already happening to you, and if they receive you badly, well no [sic]. Instead, here you come and you are received with a smile and you leave here with a better view of life, seriously. (BF-Spanish)

\section{What Are Mothers' Perceptions} of the Experience of Being an FF Mother in WIC, and How Might These Perceptions Affect

\section{Retention?}

Table 2 summarizes themes and subthemes that emerged from mothers' discussions of their perceptions of the experience of being an FF mother within WIC. Major themes included perceptions of (1) feeling judged for not BF, (2) WIC as a formula provider, (3) difficulties receiving the desired amount of formula, and (4) similar treatment of FF and BF mothers within WIC.

Feeling judged for not BF. A predominant theme that emerged within the English-speaking focus groups was a perception that FF mothers felt judged by WIC staff for not BF. Comments from several mothers reflected a perception that they had valid reasons for

not BF, but that the WIC staff did not fully understand these reasons and still encouraged them to BF, which increased their feelings of guilt for not BF. For example, 1 mother explained:

With my son, I breastfed up until about 5 months. ... I felt judged once I had to switch over to formula. And even the WIC people would ask if I was breastfeeding and I was like 'ah, I can't, like nothing is coming out' and they would be like 'well have you tried this?' I tried everything. ... I was just like 'I can't, I stopped' and because WIC is very big on breastfeeding, that it made me feel like I wasn't doing my job ... during those times, I felt very judged." (BF-English)

Several mothers also expressed a desire for WIC staff to accept their feeding choices and provide support regardless of what their choices were. As 1 mother explained:

\begin{abstract}
Like maybe identifying with us, because we do meet during the pregnancy, identifying with us what our choice is going to be, educating us throughout it obviously, like letting us know "breastfeeding is important" although "if you don't go that route, then what would you then do? Would you try do both? Or at least try to do both?" Encourage that, but then I think once we've come to the conclusion of what it's going to be.... Like I said from my experience I wanted to, I wanted to so bad but it just didn't work that way. So, to keep having to talk about it over and over every time I came in, it was just like an emotional roller coaster. (BF-English)
\end{abstract}

Many mothers indicated that the experience of feeling judged for their feeding choices made them feel out of place at WIC and reluctant to come to their WIC visits. This perception was supported by statements such as:

\footnotetext{
...when I went to my appointments I felt like "oh, my God, there she goes again." (FF-English)

Yeah because if I feel out of place, I am an adult, I don't have to come back. (BF-English)
}

It might be going back to our experiences. If we feel like we are being judged, and it could be a mental thing, someone may not be verbalizing it, but if we feel guilty in our own minds that we couldn't do the best that we could or we feel like we are not doing the best that we could, it's kind of like "you know what, I'll just avoid all of that altogether and not come back." (FF-English)

The WIC program as a formula provider. Another predominant theme that emerged within all focus groups was the idea that FF mothers primarily perceive WIC as a formula provider and that the other benefits WIC provided were not valuable enough on their own to merit continued WIC participation after the first year postpartum. A subtheme that emerged focused on cost-benefit analysis by FF mothers in that FF mothers did not think continued participation after 1 year was worthwhile given the relatively lower cost of foods (eg, milk, vegetables) compared with the expense of formula. As explained by 1 mother:

\section{And then a gallon of milk you can buy for $\$ 2$ to $\$ 3$, so they're like "why am I going to go get the checks for just a gallon of milk or whatever." That's just how I see it because I've heard people that I know say "I'm not going to go over there, I don't need the milk any- more, I already went through the formula." (FF-English)}

A related subtheme focused on the perception that $\mathrm{BF}$ mothers received more social and food support than FF mothers. Thus, once mothers stop receiving formula, they might not realize or appreciate the social and food benefits that WIC could provide to them. This phenomenon was illustrated by 1 BF mother's experience with an FF relative:

Well, my cousin ... 1 day she came with me and she noticed everything that I was getting and she was like "Wow, you get a lot. I only get formula, so I'm probably not going to come back once she's done with the formula." I think she just thought that she was out of place I guess. (BF-English) 
Table 2. What Are Mothers' Perceptions of the Experience of Being an FF Mother in WIC and How Might These Experiences Affect Retention?

Themes

Feel judged for not BF

$\mathrm{n} / \mathrm{a}$

Subthemes

WIC as formula provider

Perceived difficulties obtaining desired amount of formula
Not worth it to just get food after 12 mo

Perceive that BF moms receive more social and food support

BF-English BF-Spanish FF-English FF-Spanish

BF-English BF-Spanish FF-English FF-Spanish BF-English early on

Difficulties receiving desired amount of formula

BF-Spanish FF-English
Focus Group

BF-English

FF-English

\section{Key Examples}

.. "No, you can pump more" and it's like "No, I know my body, I'm done, I can't." Breastfeeding is not for everybody, that's just a fact. I would have loved to breastfeed because that's something nice and just more helpful, it just didn't happen ... yeah, it was just where they wanted to use the guilt card. And I understand that that's their job, but you have to understand too that if we are not able to, then we are not able to. Like you guys say, "you guys have the option of doing either or" but when they are doing this, it doesn't seem like they are giving you the option of doing it. (FF-English)

I think it is because those of us who breastfeed need it and what they give us like vegetables, eggs, cheese, to continue feeding and reproducing milk. And those who just give formula, it's only for 1 year and then they get the whole milk so maybe it makes them think it's more practical to buy the milk than to come waste time, let's say. Why bring her for just fruits and vegetables. (BF-Spanish)

I think that's what it is; I think the breastfeeding people get more and formula get less so they just don't want to continue with the whole process. (BF-English)

The staff is reluctant to give formula. Again, because when we choose to formula feed the baby, it's our personal option, right? Although we know the goodness of the breast milk, we still choose to formula-feed, it's our option. Maybe WIC can support our personal option if we chose to formula feed. Even though we know the goodness of the breast milk but we cannot give it. So, we have to have options. (BF-English) FF-Spanish

BF-English FF-English FF-Spanish
.. I did see the change that you guys give less formula. Because 11 years ago that I had my other kid they would give me more formula and instead of taking formula they added towards the end, and that really helped. And now, of course I appreciate the help, but now I have to buy more because since she's getting older we have to give more ounces. It's not so much of giving them more formula, it's just more ounces that we do give the baby, so it goes way faster. Because I go through a can in like 3 days, because I do give her 6-8 oz in 1 feeding. (FF-English) They don't treat you different; they just try to motivate you to breastfeed. (FF-English)

mothers within WIC

BF indicates breastfeeding; FF, formula-feeding; n/a, not applicable; WIC, Special Supplemental Nutrition Program for Women, Infants, and Children. 
Difficulty receiving desired amount of formula. In all focus groups, some discussion focused on the difficulties that mothers experienced in receiving formula early on or receiving the amount of formula they thought they needed to feed their infant. With respect to difficulties in receiving formula early on, several mothers within the BFEnglish group recounted that the staff was reluctant to provide them with formula shortly after their infant's birth despite their perceived need for formula because of BF problems. With respect to receiving their desired amount of formula, mothers all expressed dissatisfaction with their perception that the WIC packages did not provide enough formula for their older infants (aged $>6$ months). Many mothers indicated that they typically had to buy 1-2 additional cans of formula per month with their own money.

Similar treatment of FF and BF mothers within WIC. A few mothers within the BF-English, FF-English, and FFSpanish focus groups did not think that WIC treated FF mothers differently than BF mothers. They acknowledged that WIC was supportive of BF, but also perceived that FF mothers were supported.

\section{DISCUSSION}

Participation in the WIC program has declined $17 \%$ nationwide over the past 4 years. $^{25}$ In addition, a recent report illustrated that of the eligible families participating in WIC, 23\% exited the program when their infant turned 1 year old. ${ }^{10}$ Previous research illustrated that mothers who were fully FF their infants from age 6-12 months were 3 times less likely to recertify for WIC at age 1 year compared with mothers who were fully BF their infants during this same period. ${ }^{15}$ Given these findings, the aim of the current study was to better understand mothers' perceptions of why propensity to recertify in WIC may differ between BF and FF mothers. To date, few studies have examined associations between mothers' feeding choices and experiences within WIC, which has left a critical gap in understanding of why FF mothers are less likely to recertify their children at age 1 year.

Mothers in the WIC program recognized and discussed several important benefits of WIC, including BF support, relevant nutrition education tailored to their families' developing needs, and financial assistance through the provision of formula and foods. These benefits highlight key reasons why mothers value WIC and potential motivators for continued participation in the program. With respect to BF support, 1 of the hallmarks of the WIC program is its robust BF promotion and support aimed at facilitating WIC mothers' abilities for successful BF. This support comes in many forms, including early and frequent assessment of BF mothers' struggles and needs by WIC staff, availability of in-person and online BF education, access to BF peer counselors and BF clinics in some sites, and BF support warm lines. The need for and utility of these BF resources is supported by previous research illustrating how strong social support was an important foundation for the initiation and successful continuation of $\mathrm{BF}$ among both WIC and non-WIC mothers. ${ }^{26-31}$ Research showed that postpartum WIC mothers who received professionally mediated peer support, such as WIC peer counselors, were exclusively BF for significantly longer durations compared with women who did not receive special BF support. ${ }^{28}$ In addition, women who received $\mathrm{BF}$ peer support reported significantly greater satisfaction with BF. In general, the combination of $\mathrm{BF}$ information with affirmation and encouragement of BF efforts is an important social support action associated with longer BF duration and satisfaction. ${ }^{28}$

Despite recognition of the multitude of ways in which WIC supports both BF and FF mothers, focus group discussions revealed differences in the type of resources allocated to BF vs FF mothers during the first year postpartum. Indeed, WIC provides substantial material support to all WIC mothers by providing food packages and other services. ${ }^{32,33}$ However, an analysis conducted by the National Academies of Sciences, Engineering, and Medicine ${ }^{32}$ estimated that the
2015 market (retail) values of the FF packages were substantially higher than the market values of the $\mathrm{BF}$ packages. This difference was partly explained by the fact that BF WIC mothers received more in services that provided social support for $\mathrm{BF}^{32}$; however, no comparable equivalent is provided to FF WIC mothers. Differences in resource allocation for WIC mothers depending on their chosen infant-feeding method has been a long-standing concern given that WIC's provision of formula may remove a powerful economic incentive to $\mathrm{BF} .^{5}$ In addition, provision of formula may create a strong financial incentive for FF mothers to participate in WIC through the first year postpartum, whereas BF mothers may be more motivated to participate in WIC because of both the financial and social incentives provided by the program.

According to self-determination theory, extrinsic incentives (eg, money) are stronger predictors of short-term behavior than intrinsic incentives (eg, social connectedness), especially when the extrinsic incentives are in place and available. ${ }^{34}$ However, extrinsically motivated behaviors are not sustainable because they typically cease once the incentives are removed and are associated with lower valuation of the behavior, because it is strongly connected with external worth indicators. ${ }^{35,36}$ Thus, promotion of intrinsically motivated behaviors is more desirable because these behaviors typically persist longer and at a higher level of performance, which in turn fosters sustainable behavior change. ${ }^{34,37,38}$ Key predictors of intrinsic motivation are social supports that promote autonomy, competence, relatedness, and self-efficacy. $^{34,39}$ When considering focus group findings within the selfdetermination theory framework, it is possible that FF mothers who predominantly receive material supports from WIC were less motivated to stay in the program after formula benefits ceased, whereas BF mothers who received both material and social supports felt more connected to the WIC program and motivated to continue using WIC services even when material supports declined. 
In further support of this possibility, focus group participants also discussed the idea of a cost-benefit decision FF mothers may make once formula benefits cease, in which the benefits provided by WIC may no longer seem worth the effort expended to obtain those benefits. Examples of similar cost-benefit decisions among WIC participants were illustrated in previous research in which WIC families reported that the ongoing burdens of WIC requirements (eg, inperson appointments) outweighed the benefits of receiving food packages. ${ }^{40}$ Moreover, there is evidence that the perceived benefits of a program affect an individual's decision to participate in that program; specific to WIC, the market value of the FF package is considerably higher than that of the BF package, ${ }^{32}$ which suggests that this benefit (eg, the FF package) outweighs the cost (eg, the effort needed to come to WIC). ${ }^{12}$ In other words, the FF package is deemed valuable, and thus keeps FF moms in the WIC program through the first year. However, once this benefit ceases at the child's first birthday, FF mothers may no longer see the value in the program.

A concerning theme that emerged within the focus groups was the perception that some FF mothers felt judged for their choice not to breastfeed. This perception was consistent with previous research illustrating how, in many cultures, BF is associated with good and adequate mothering. ${ }^{41-43}$ Thus, when mothers do not breastfeed, feelings of guilt, shame, blame, and failure may arise $\mathrm{e}^{44,45}$ owing to perceived failure to fulfill the expectations of motherhood. ${ }^{46}$ Many FF women report feeling judged for not BF within their social networks and, more often, with health professionals. ${ }^{47}$ Health professionals' disapproval of FF may have led them to avoid talking about or offering support for $\mathrm{FF}^{47}$ especially if this was viewed as a threat to supporting $B F$. In fact, some argued that the focus on increasing $\mathrm{BF}$ rates led to the marginalization and isolation of $\mathrm{FF}$ mothers ${ }^{43,44}$ and to a disconnect between the needs of FF mothers and the focus of health professionals. ${ }^{43}$ Indeed, previous qualitative research illustrated that FF mothers reported receiving inadequate support for learning healthy bottle-feeding practices from health care providers, partially because greater emphasis was placed on $\mathrm{BF}^{44}$ Formula-feeding mothers also reported a lack of confidence in the appropriateness of their bottle-feeding behaviors because they had to rely on their own judgment or nonprofessional sources of information. ${ }^{44}$ This problem is accentuated in low-income, minority populations who have higher rates of FF, greater risk for using problematic bottle-feeding practices, and are more likely to seek friends and family, instead of professionals, for infantfeeding advice. ${ }^{48,49}$ Thus, FF mothers' feelings of stigma and judgment are especially concerning given the high prevalence of FF among WIC populations. These findings suggest that expansion of WIC counseling and programming to create a more inclusive and welcoming environment for all mothers would continue to provide resources to $\mathrm{BF}$ mothers, but might also enhance social support for FF mothers and potentially encourage WIC participation well into the preschool years for all qualified children.

Finally, the themes that emerged related to mothers' perceived inadequacy of formula provisions and the limited issuance of formula in the first month postpartum are important to address. With respect to the perceived inadequacy of formula provisions, the FF package implemented by the US Department of Agriculture in 2009 calibrated formula amounts to meet nearly $100 \%$ of the caloric needs of the FF infant from birth through age 5 months, with increased amounts at 4-5 months as caloric needs increase. ${ }^{9}$ This effectively means that for FF infants aged less than 6 months, WIC provides all of the nutrition needed and mothers should not need to purchase additional formula to meet their infants' caloric needs. It is not clear whether this is well-known by WIC participants or staff, which highlights the need for expanded training for staff and targeted nutrition education to reduce overfeeding and/or formula waste for mothers with FF infants aged less than 6 months and who perceive the need to purchase additional formula with their own funds. In particular, this targeted nutrition education could focus how to be responsive to infant hunger and satiation cues during bottle-feeding so that infants are not given too much formula and/or expected to empty the bottle at every feed (for example, paced bottle-feeding ${ }^{50}$ ). It is also possible that mothers of older FF infants are not providing enough complementary foods and beverages to meet their infants' needs; thus, nutrition education about how much infants need at each developmental stage is essential for all WIC infants.

With respect to perceived limitations regarding formula issuance in the first month after birth, WIC federal rules about issuing formula in the first month postpartum were examined and room for improvement was suggested. This was in fact a topic that received significant attention from the recent National Academy of Sciences, Engineering, and Medicine Committee to review WIC Food Packages. ${ }^{32} \mathrm{~A}$ subsequent commentary by members of the committee highlighted the intention of the recommendations to encourage increased flexibility in determining the amount of formula offered to partially BF infants in the first month. ${ }^{51}$ Findings from the current study provide further evidence that changes in policy regarding issuance of formula in the early postpartum period may help FF mothers feel more supported by WIC.

A strength of this study was the use of focus groups and qualitative methods to explore an understudied issue that is relevant to the WIC population. Findings from this study are an important foundation for further qualitative and quantitative research aimed at understanding better how to continue to improve WIC recruitment and retention efforts. Limitations of this study highlight opportunities for this future research. Key limitations of this study were the small sample size and the limited demographic composition of the focus groups. The study included only 31 WIC mothers from 2 targeted WIC sites, and they were either white or Hispanic. In addition, because focus groups were organized by feeding mode (BF vs FF) and language spoken (English vs Spanish), it is possible that these groups were not equivalent based on their feeding and cultural experiences, and that these 
findings might have been broadened if a greater number of focus groups had been conducted. Future research including larger samples with participants from a wider array of sites, races, and ethnicities, as well as focus groups with WIC personnel, is warranted. In addition, participants were from a large metropolitan area of southern California; hence, results may not be generalizable or transferable to other WIC populations.

\section{IMPLICATIONS FOR RESEARCH AND PRACTICE}

The aims of this exploratory focus group study were to examine the ways in which WIC supports both BF and FF mothers and to understand how mothers' experience of being an FF mother in WIC might influence continued participation in the program. An important theme that emerged was that BF women felt that there was strong support from WIC, but FF mothers often felt underserved with respect to support for their feeding decisions, and some thought that they were judged for their choice of FF rather than BF. Thus, although WIC provides abundant and varied $\mathrm{BF}$ support, it may be important to incorporate more robust social support and hands-on education to FF mothers as well. A striking paucity of studies has focused on improving the feeding practices of FF mothers during early infancy; thus, more research is needed to understand how best to support FF mothers in a way that does not compromise BF. Given the ubiquity of FF, especially among WIC populations, this evidence base will be a critical step toward ensuring that all WIC participants, regardless of whether they are $\mathrm{BF}$ or $\mathrm{FF}$, remain in the program for as long as they are eligible and receive the full array of benefits that WIC has to offer.

\section{ACKNOWLEDGMENTS}

Funding for the focus groups conducted was provided by a grant from the State of California WIC program to the Public Health Foundation Enterprises WIC program. The authors would like to acknowledge Martha Meza, Elizabeth Rodriguez, Amanda Herrera, Sharen Anthony, Cindy
Clapp, Judy Gomez, Kiran Saluja, Lizbeth Delatorre, May Wang, and WIC participants for their support of and participation in this project. They also thank Lily Hawkins and Jessi Modlich for their assistance with the qualitative analysis of the focus group transcripts.

\section{REFERENCES}

1. U.S. Department of Agriculture Food and Nutrition Service. WIC's mission. https://www.fns.usda.gov/wic/aboutwic-wics-mission. Accessed October 18, 2019.

2. U.S. Department of Agriculture Food and Nutrition Service. Frequently asked questions. https://www.fns.usda. gov/wic/frequently-asked-questionsabout-wic. Accessed October 18, 2019.

3. U.S. Department of Agriculture, Economic Research Service. WIC program. https://www.ers.usda.gov/topics/foodnutrition-assistance/wic-program.aspx. Accessed October 18, 2019.

4. Liu CH, Liu H. Concerns and structural barriers associated with WIC participation among WIC-eligible women. Public Health Nurs. 2016;33: 395-402.

5. Bitler MP, Currie J. Does WIC work? The effects of WIC on pregnancy and birth outcomes. J Policy Anal Manag. 2005;24:73-91.

6. Gai F, Feng L. Effects of federal nutrition program on birth outcomes. Atl Econ J. 2012;40:61-83.

7. Nestor B, McKenzie J, Hasan N, AbuSabha R, Achterberg C. Client satisfaction with the nutrition education component of the California WIC program. J Nutr Educ. 2001;33:83-94.

8. Owen AL, Owen GM. Twenty years of WIC: a review of some effects of the program. J Am Diet Assoc. 1997;97: 777-782.

9. Special Supplemental Nutrition Program for Women, Infants, and Children (WIC). Revisions in the WIC food packages, 7 C.F.R. $\S 46.101$ (2014). Final rule. https://www.fns.usda.gov/wic/fr030414. Accessed January 2, 2020.

10. Jacknowitz A, Tiehen L. WIC participation patterns: an investigation of delayed entry and early exit. Washington, DC: US Department of Agriculture, Economic Research; 2010. https://www. ers.usda.gov/publications/pub-details/? pubid $=44783$.
11. Sekhobo JP, Peck SR, Byun Y, et al. Use of a mixed-method approach to evaluate the implementation of retention promotion strategies in the New York State WIC program. Eval Program Plann. 2017;63:7-17.

12. Martin-Anderson S. Prenatal attitudes and parity predict selection into a U.S. child health program: a short report. Soc Sci Med. 2013;95:128-132.

13. Gilbert D, Nanda J, Paige D. Securing the safe net: concurrent participation in income eligible assistance programs. Matern Child Health J. 2014;95:128-132.

14. Kahler LR, O'Shea RM, Duffy LC, Buck GM. Factors associated with rates of participation in WIC by eligible pregnant women. Public Health Rep. 1992;107:60-65.

15. Whaley SE, Whaley M, Au LE, Gurzo $\mathrm{K}$, Ritchie LD. Breastfeeding is associated with higher retention in WIC after age 1. J Nutr Educ Behav. 2017;49:810816.

16. Department of Health and Human Services. Protection of Human Subjects, 45 C.F.R. $\$ 46.101$ (2009). Final Rule.

17. Guest G, Namey E, McKenna K. How many focus groups are enough? Building an evidence base for nonprobability sample sizes. Field Methods. 2016;29:3-22.

18. Krueger RA, Casey MA. Focus Groups: A Practical Guide for Applied Research. Thousand Oaks, CA: Sage Publications; 2015.

19. Kim LP, Koleilat M, Whaley SE. A qualitative study to examine perceptions and barriers to appropriate gestational weight gain among participants in the special supplemental nutrition program for women infants and children program. $J$ Preg. 2016;2016:4569742.

20. Montaño DE, Kasprzyk D. Chapter 6 Theory of reasoned action, theory of planned behavior, and the integrated behavioral model. In: Glanz K, Rimer BK, Viswanath K, eds. Health Behavior: Theory, Research, and Practice, 5th ed. San Francisco, CA: Jossey-Bass; 2015:95-124.

21. Burnard P, Gill P, Stewart K, Treasure E, Chadwick B. Analysing and presenting qualitative data. Br Dent J. 2008; 204:429-432.

22. Charmaz K. Grounded theory: objectivist and constructivist methods. In: Denzin K, Lincoln YS, eds. Handbook of Qualitative Research, Thousand Oaks, CA: Sage; 2000:509-536.

23. Harry E, Sturges KM, Klingner JK. Mapping the Process: an exemplar of 
process and challenge in grounded theory analysis. Educ Res. 2005;34:3-13.

24. Korstjens I, Moser A. Series: practical guidance to qualitative research. part 4: trustworthiness and publishing. Eur $J$ Gen Pract. 2018;24:120-124.

25. US Department of Agriculture, Food and Nutrition Service. WIC Data Tables. 2019. https://www.fns.usda.gov/pd/ wic-program. Accessed January 2, 2020.

26. Oniwon O, Tender JA, He J, Voorhees E, Moon RY. Reasons for infant feeding decisions in low-income families in Washington, DC. J Hum Lact. 2018;32: 704-710.

27. Dunn RL, Kalich KA, Fedrizzi R, Phillips S. Barriers and contributors to breastfeeding in WIC mothers: a social ecological perspective. Breastfeed Med. 2015;10:493-501.

28. Vari PM, Camburn J, Henly S. Professionally mediated peer support and early breastfeeding success. J Perinat Educ. 2000;9:22-30.

29. Duckett 1, Henly SJ, Garvis M. Predicting breast-feeding duration during the postpartum hospitalization. West J Nurs Res. 1993;15:177-198.

30. Matich JR, Sims LS. A comparison of social support variables between women who intend to breast or bottle feed. Soc Sci Med. 1992;34:919-927.

31. Raj VK, Plichta SB. The role of social support in breastfeeding promotion: a literature review. J Hum Lact. 1998;14: 41-45.

32. National Academies of Sciences, Engineering, and Medicine. Review of WIC Food Packages: Improving Balance and Choice: Final Report. Washington, DC: The National Academies Press; 2017.

33. Carlson S, Neuberger Z, Rosenbaum D. WIC participation and costs are stable: have returned to pre-recession levels. Washington, DC: Center on Budget and Policy Pri- orities; 2017. https://www.cbpp.org/ sites/default/files/atoms/files/8-3-15fa. pdf. Accessed October 18, 2019.

34. Deci EL, Ryan RM. The "what" and "why" of goal pursuits: human needs and the self-determination of behavior. Psychol Inq. 2000;11:227-268.

35. Vansteenkiste M, Simons J, Soenens B, Lens W. How to become a persevering exerciser? Providing a clear, future intrinsic goal in an autonomy-supportive way. J Sport Exerc Psychol. 2004; 26:232-249.

36. Stockdale J, Sinclair M, Kernohan G, Lavender $\mathrm{T}$. What do we mean when we talk about intrinsic and extrinsic motivation to breastfeed? A commentary. J Hum Lact. 2010;26:15-17.

37. Vansteenkiste M, Simons J, Lens W, Sheldon KM, Deci EL. Motivating learning, performance, and persistence: the synergistic effects of intrinsic goal contents and autonomy-supportive contexts. J Pers Soc Psychol. 2004;87:246-260.

38. Teixeira PJ, Silva MN, Coutinho SR. al. Mediators of weight loss and weight loss maintenance in middle-aged women. Obesity (Silver Spring). 2010;18:725-735.

39. Grolnick WS, Deci EL, Ryan RM. Internalization within the family: the self-determination theory perspective. In: Grusec JE, Kuczynski L, eds. Parenting and Children's Internalization of Values: A Handbook of Contemporary Theory, New York: Wiley; 1997:135-161.

40. Pati S, Siewert E, Wong AT, Bhatt SK, Calixte RE, Cnaan A. The influence of maternal health literacy and child's age on participation in social welfare programs. Matern Child Health J. 2014;18: 1176-1189.

41. Schmied V, Barclay L. Connection and pleasure, disruption and distress: women's experience of breastfeeding. J Hum Lact. 1999;15:325-334.
42. Hauck YL, Irurita VF. Constructing compatibility: managing breast-feeding and weaning from the mother's perspective. Qual Health Res. 2002;12: 897-914.

43. Dykes F, Flacking R. Encouraging breastfeeding: a relational perspective. Early Hum Dev. 2010;86:733-736.

44. Lakshman R, Ogilvie D, Ong KK. Mothers' experiences of bottle-feeding: a systematic review of qualitative and quantitative studies. Arch Dis Child. 2009;94:596-601.

45. Lee E. Health, morality, and infant feeding: British mothers' experiences of formula milk use in the early weeks. Sociol Health Illn. 2007;29:1075-1090.

46. Taylor EN, Wallace LE. For shame: feminism, breastfeeding advocacy, and maternal guilt. Hypatia. 2011;27:76-98.

47. Thomson G, Ebisch-Burton K, Flacking R. Shame if you do - shame if you don't: women's experiences of infant feeding. Matern Child Nutr. 2014;11: 33-46.

48. Gross RS, Mendelsohn AL, Fierman AH, Hauser NR, Messito MJ. Maternal infant feeding behaviors and disparities in early child obesity. Child Obes. 2014; 10:145-152.

49. Heinig MJ, Ishii KD, Banuelos JL, Campbell E, O'Loughlin C, Vera Becerra LE. Sources and acceptance of infantfeeding advice among low-income women. J Hum Lact. 2009;25:163-172.

50. Kassing D. Bottle-feeding as a tool to reinforce breastfeeding. J Hum Lact. 2002;18:56-60.

51. Rasmussen KM, Whaley SE, PerezEscamilla R, et al. New opportunities for breastfeeding promotion and support in WIC: review of WIC food packages, improving balance and choice. J Nutr Educ Behav. 2017;49(suppl 2):S197S201. 